\title{
Super-heavy Quarkonia and the Gluon Condensate
}

\author{
Jochen Fingberg \\ University of Wuppertal and CCRL, NEC Europe Ltd., 53757 St.Augustin, Rathausallee 10, Germany
}

The early idea that a non-perturbative gluon condensate affects the spectrum of heavy quarks is revisited in the light of modern simulation techniques. We evaluate the low lying spectrum of bound states of two heavy quarks for large hypothetical quark mass, $m_{Q}>m_{b}$, using non-relativistic QCD and compare with other models to test the consistency.

The concept of a non-perturbative gluon condensate was initially introduced by Shifman, Vainstein, and Zakharov [1]. A non-vanishing gluon condensate can give rise to a shift of energy levels of heavy quarkonia by second order Stark splitting. Leutwyler and Voloshin [2] calculated the level shifts in leading order perturbation theory. Recent improvements like the stochastic vacuum model SVM [0], non-perturbative field strength correlators [3] or a combination of the SVM with lattice calculations [6,7]) try to solve some of the problems of the original LV-model. Using NRQCD has the advantage that no apriori knowledge about the heavy quark potential or the vacuum background field is needed. The physical problem is reduced to a simulation of non-relativistic heavy quarks in a correlated, relativistic gluonic background. We use an $\mathcal{O}\left(a^{4}\right)$ improved gauge action with a plaquette and a $1 \times 2$ Wilson loop term for the relativistic simulations and an $\mathcal{O}\left(v^{4}, v^{6}\right)$ corrected non-relativistic action

$S_{N R}=\psi^{\dagger}\left(D_{t}+H_{0}+\delta H\left(c_{i}\right)\right) \psi$

with tree level couplings, $c_{i}=1$, for the heavy quarks. The quark's Green's functions are calculated from the standard (asymmetric in $\delta H$ ) evolution equation. Meson propagators with specific quantum numbers are obtained from the quark propagators using a smearing procedure with trial wave-functions from a solution of the radial Schrödinger equation using a method from ref. 13 for each value of the bare quark mass. Preliminary results based on Coulomb wavefunctions and a small subset of the configurations were already reported at a previous conference
10].

For the simulations we use a lattice of size $16^{4}$ and a gauge coupling of $\beta=9.17$ to obtain a very fine spacing necessary to resolve small bound states. After generating 360 decorrelated configurations on a Quadrics Q4 we fix to Coulomb gauge with a stopping criterion, $\theta<10^{-6}$. The simulations require 60 days of Quadrics Q4 time, 70 days on a workstation for gauge-fixing, and more than 150 days of CM2/CM5 time. We apply standard correlated multi-state multi-exponential fits with vector fits for a set of $\mathrm{S}$ states and ratio fits for hyperfine and S-P splittings. The error is determined by a jackknife procedure including the uncertainty from the fit range, $\left[t_{\min }, N_{\tau}\right]$. A consistent scale is obtained from $V_{q \bar{q}}$ and the force indicating that scaling violations are indeed small at this high value of the gauge coupling (see ref. 15. for details).

As a first step our results can be compared to leading order perturbation theory which allows to separate the Coulomb from the condensate contribution. Following refs. 89,9] the level splittings are given to lowest order by the expressions

$$
\begin{aligned}
\Delta E_{h f s} & \simeq \frac{m\left(C_{F} \alpha_{s}\right)^{4}}{3}+\frac{.5425 \pi C_{F}^{4}}{m^{3} \alpha_{s}^{2}} f_{10}\left\langle\alpha_{s} G^{2}\right\rangle \\
\Delta E_{S P} & \simeq \frac{m\left(C_{F} \alpha_{s}\right)^{2}}{16 / 3}+\frac{64 \pi}{C_{F}^{4} m^{3} \alpha_{s}^{4}} f_{21}\left\langle\alpha_{s} G^{2}\right\rangle \\
f_{n l}^{-1} & =1+\left(\lambda_{G}^{-1} /\left[m\left(4 / 3 \alpha_{s}\right)^{2}\right]\right) \rho_{n l} \\
\rho_{10} & =2.48, \quad \rho_{21}=11.20, \quad C_{F}=4 / 3 .
\end{aligned}
$$

There are two length scales in the system: the gluon correlation length $\lambda_{G}$ and the quark correlation length $\lambda_{Q}$ which is related to the rotational period of the two heavy quarks inside the quarko- 


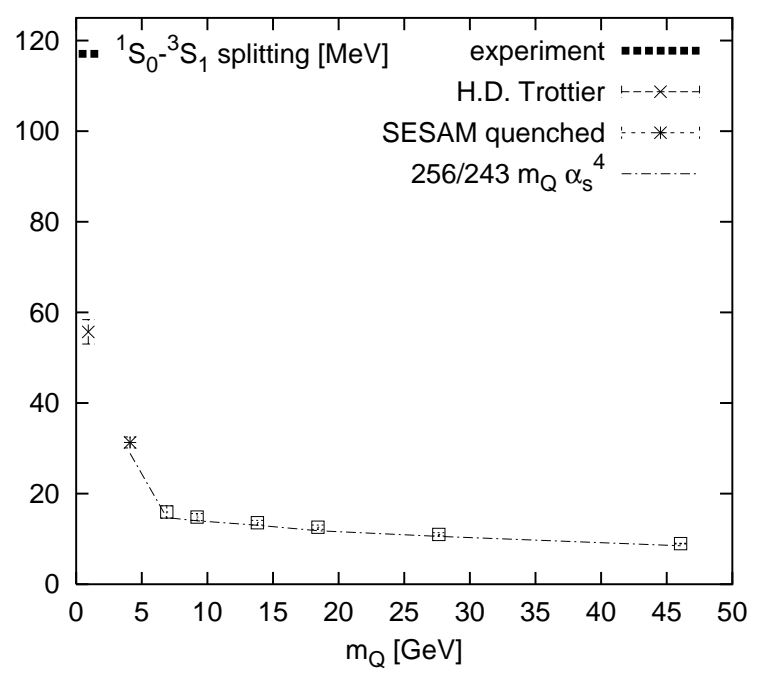

Figure 1. The hyperfine splitting between the ground state of the ${ }^{3} S_{1}$ and the ${ }^{1} S_{0}$ meson. The line gives the perturbative Coulomb contribution with $\alpha_{s}\left(m_{Q}\right)$ as shown in fig. 3. Also shown are the $c \bar{c}$ [11] and $b \bar{b}$ values [12].

nium, $\lambda_{Q} \simeq 2 \pi \sqrt{\left\langle r^{2}\right\rangle /\left\langle v^{2}\right\rangle}$. The shortest correlation length governs the behaviour of the energy levels. There are two limiting cases: a static condensate $\left(\lambda_{G}>>\lambda_{Q}\right)$ when $f_{n l}=1$ and a rapidly varying condensate $\left(\lambda_{G}<<\lambda_{Q}\right)$ when $f_{n l}<1$.

We can solve the set of equations $\left\{\Delta E_{h f s}\right.$, $\left.\Delta E_{S P}\right\}$ for the two unknowns $\alpha_{s}$ and $\left\langle\alpha_{s} G^{2}\right\rangle$ inserting the values for the splittings from NRQCD shown in figs. 1 and 2. In this way we obtain the strong coupling and the condensate as a function of the bare quark mass shown in figs. 3 and 4 . If we reverse this procedure for a while and accept that fig. 3 describes a reasonable behaviour of the running coupling $\alpha_{s}\left(m_{Q}\right)$ then the perturbative Coulomb contribution $\left(\simeq 1.05 m_{Q} \alpha_{s}^{4}\right)$ shown in fig. 1 as a line together with our data completely dominates the hyperfine splitting $E_{h f s}$. On the other hand for the S-P splitting the condensate contribution is larger than the Coulombic part which has been criticised before and sheds doubt on the treatment of the condensate as a perturbation. The magnitude of the contributions to $E_{h f s}$ and $E_{S P}$ does not change significantly as the gluon condensation length is varied. The parameter $\lambda_{G}$ merely rescales the value of the

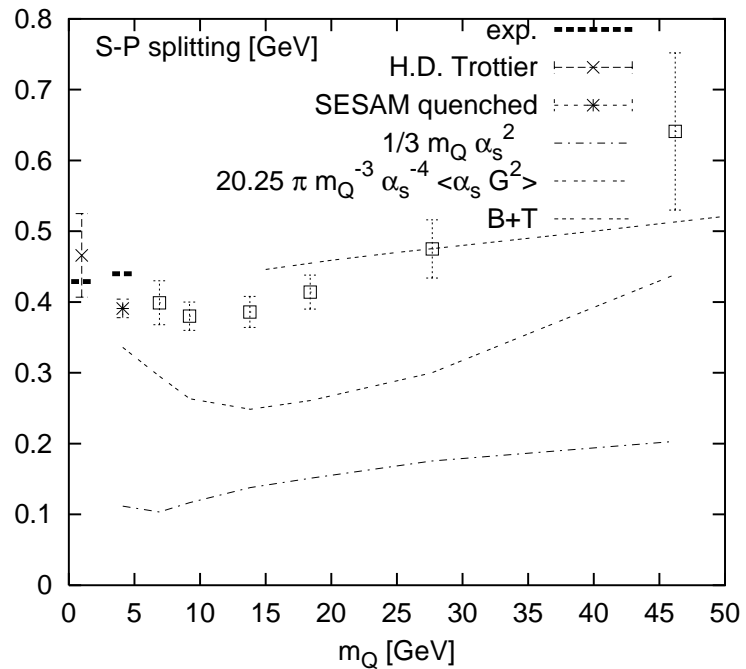

Figure 2. The S-P splitting from NRQCD (squares), perturbation theory and for the Buchmüller and Tye model 低.

condensate by a factor $f_{n l}\left(\lambda_{G}\right)$. If we nevertheless take the perturbative results seriously we see from fig. 4 that it is possible to get a value of the condensate compatible with sum rule results. However, the value of $\left\langle\alpha_{s} G^{2}\right\rangle$ strongly varies with quark mass. The static approximation $\left(\lambda_{G} \rightarrow \infty\right)$ should be valid for large quark mass while the approximatiuon of a rapidly varying condensate $\left(\lambda_{G}<<\lambda_{Q}\right)$ is expected to hold for intermediate masses, $m_{Q}<40 \mathrm{GeV}$, because $\lambda_{Q}$ is decreasing with $m_{Q}$.

\section{Summary}

At large $m_{Q}$ the hyperfine splitting of the ground state becomes compatible with Coulombic behaviour. However, the S-P splitting from NRQCD is neither in accord with the $\mathrm{B}+\mathrm{T}$ model nor with leading order perturbative results for LV-type models. On the level of lowest order perturbation theory no consistent picture of the effect of the gluon condensate can be found allthough there is evidence that the general trend predicted by the analytic results is qualitatively correct. The NRQCD hyperfine splitting of the ground state is decreasing and the S-P splitting is increasing with $m_{Q}$ for large quark mass consistent with the perturbative dependence on dif- 


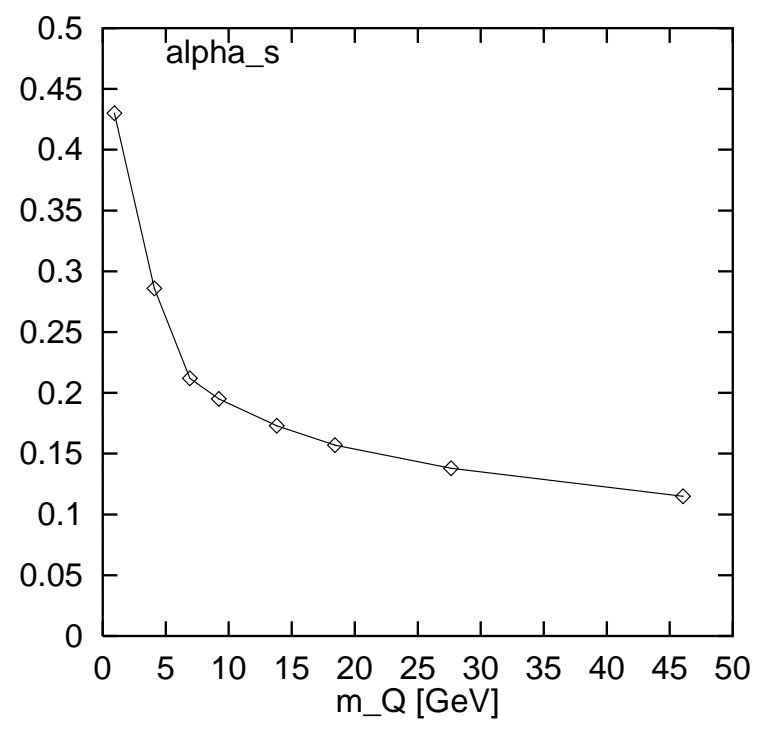

Figure 3. The running coupling $\alpha_{s}$ from a comparison of the S-P and the hyperfine splitting of the ground state from NRQCD with perturbative predictions.

ferent powers of $\alpha_{s}$. It will be interesting to see 15 if a comparison with more rigorous models like the combined SVM and lattice approach will consolidate the situation. An alternative might be a closed lattice approach. Then one is faced with the difficult problem to switch the condensate on and off during the simulations.

\section{Acknowledgement}

J.F. was supported by a DFG fellowship during the initial phase of the project. We also wish to thank the IAI at the University of Wuppertal and the computer centre of the University of Erlangen for their support.

\section{REFERENCES}

1. V.I. Zakharov, (1978); M.A. Shifman, A.I. Vainstein and V.I. Zakharov, Nucl. Phys. B147 (1979) 385 and 448.

2. M.B. Voloshin, Nucl. Phys. B154 (1979) 365; H. Leutwyler, Phys. Lett. B98 (1981) 447.

3. A. Di Giacomo and H. Panagopoulos, Phys. Lett. B285 (1992) 133.

4. W. Buchmüller and S.-H.H. Tye, Phys. Rev.

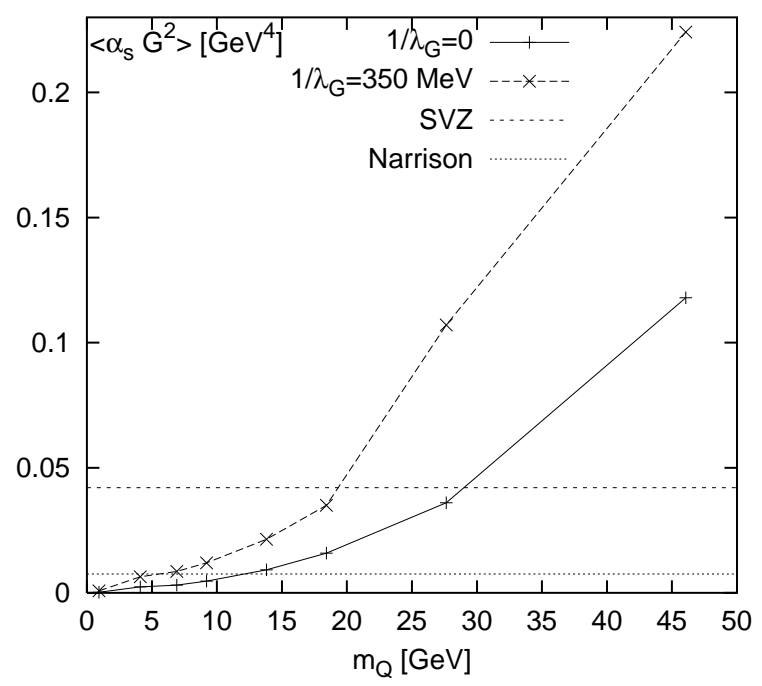

Figure 4. A comparison of the gluon condensate for the static case, $\lambda_{G} \rightarrow \infty$ and for $\lambda_{G}^{-1}=350$ $\mathrm{MeV}$ with different values from the literature (SVZ: [1, Narrison: [14]).

D24 (1981) 132; J.L. Rosner et al., Phys. Rev. D53 (1996) 2742.

5. H.G. Dosch and Yu.A. Simonov, Phys. Lett. B209 (1988) 339; Z. Phys. C45 (1989) 147.

6. G. Bali, K. Schilling and A. Wachter, Phys. Rev. D56 (1997) 2566.

7. G. Bali, N. Brambilla and A. Vairo, eprintarchive hep-lat/9709079, December 1997.

8. S. Titard and F.J. Yndurain, Phys. Rev. D49 (1994) 6007; Phys. Lett. B351 (1995) 541; Phys. Rev. D51 (1995) 6348.

9. Yu.A. Simonov, S. Titard and F.J. Yndurain, Phys. Lett. B354 (1995) 435.

10. J. Fingberg, Nucl. Phys. Proc. Suppl. 53 (1996) 405.

11. H.D. Trottier, Phys. Rev. D55 (1997) 6844.

12. N. Eicker, T. Lippert, K. Schilling, A. Spitz, J. Fingberg, S. Gusken, H. Hoeber, J. Viehoff, HLRZ-1997-35, Sep 1997.

13. A. Wachter, PhD-thesis, Universität Wuppertal, WUB-DIS 96-21, November 1996.

14. S. Narrison, Phys. Lett. B 1996.

15. J. Fingberg, work in progress. 